\title{
A nomogram to predict invasiveness in lung adenocarcinoma presenting as ground glass nodule
}

\author{
Nan Zhang ${ }^{1}$, Jun-Feng Liu ${ }^{1}$, Ya-Ning Wang ${ }^{2}$, Li Yang ${ }^{2}$ \\ ${ }^{1}$ Department of Thoracic Surgery, ${ }^{2}$ Imaging Department, Fourth Hospital of Hebei Medical University, Shijiazhuang 050000, China \\ Contributions: (I) Conception and design: N Zhang; (II) Administrative support: JF Liu; (III) Provision of study materials or patients: YN Wang; (IV) \\ Collection and assembly of data: L Yang; (V) Data analysis and interpretation: N Zhang; (VI) Manuscript writing: All authors; (VII) Final approval of \\ manuscript: All authors. \\ Correspondence to: Jun-Feng Liu. Department of Thoracic Surgery, Fourth Hospital of Hebei Medical University, 12 Jiankang Road, Shijiazhuang \\ 050000, China. Email: liujf@hbmu.edu.
}

Background: Adenocarcinoma in situ, minimally invasive adenocarcinoma, and invasive adenocarcinoma (IA) can all appear as a ground glass opacity (GGO) on chest computed tomography (CT). However, their respective prognoses are considerably different. This study aimed to predict IA in radiological examinations of patients with GGO lesions.

Methods: We retrieved the clinical records and high-resolution CT (HRCT) images of 124 patients with GGO lesions, who underwent various lung resections between 2016 and 2017. Correlations between the imaging features of preoperative HRCT and the postoperative pathology were analyzed. Receiver-operating characteristic (ROC) curve analysis, chi-square test, and one-way analysis of variance and multiple logistic regression were performed. A nomogram was developed and analyzed using a multiple logistic model.

Results: The maximum sensitivity and specificity were obtained at a cutoff value of -410 Hounsfield units (HU) for the mean CT value (m-CT), $10 \mathrm{~mm}$ for the maximum tumor dimension (MTD), and 0.25 for the consolidation tumor ratio (CTR). Further, there were significant differences in MTD, CTR, margin characteristics, air bronchogram, pleural indentation, and multiple GGOs $(\mathrm{P}<0.05)$. The independent predictive factors of IA included MTD [risk ratio (RR), 5.047; $\mathrm{P}=0.018$ ], air bronchogram or vacuole sign ( $R R$, 4.054; $\mathrm{P}=0.025)$, pleural retraction $(\mathrm{RR}, 4.742 ; \mathrm{P}=0.008)$, and $\mathrm{m}-\mathrm{CT}$ value $(\mathrm{RR}, 5.874 ; \mathrm{P}=0.005)$. The scoring nomogram model was as follows: $-3.50744+1.26374 \times(\mathrm{MTD}>10 \mathrm{~mm}=1)+2.41978 \times(\mathrm{m}-$ CT value $\geq-410 \mathrm{HU}=1)+1.77779 \times($ with air bronchogram or vacuole sign=1) $+1.60913 \times$ (with pleural retraction=1). The area under the ROC curve was 0.9 . The cutoff score was -0.5502 with a sensitivity of $86.8 \%$ and a specificity of $78.9 \%$.

Conclusions: IA in patients with GGO lesions can be predicted by evaluating the MTD, m-CT value, air bronchogram, and pleural retraction on HRCT by using a nomogram model.

Keywords: Nomogram, ground glass opacity (GGO); high-resolution computed tomography (HRCT); lung adenocarcinoma; mean CT value (m-CT value)

Submitted Oct 23, 2019. Accepted for publication Jan 07, 2020.

doi: $10.21037 /$ tcr.2020.01.55

View this article at: http://dx.doi.org/10.21037/tcr.2020.01.55

\section{Introduction}

Lung cancer is one of the most common tumors, with morbidity and mortality rates ranking in the top 3 among all cancers worldwide (1). Adenocarcinoma is the most common pathological type globally. The new pathological classification system suggested some new types such as adenocarcinoma in situ (AIS) and minimally invasive adenocarcinoma (MIA) (2). The invasive status of a tumor 


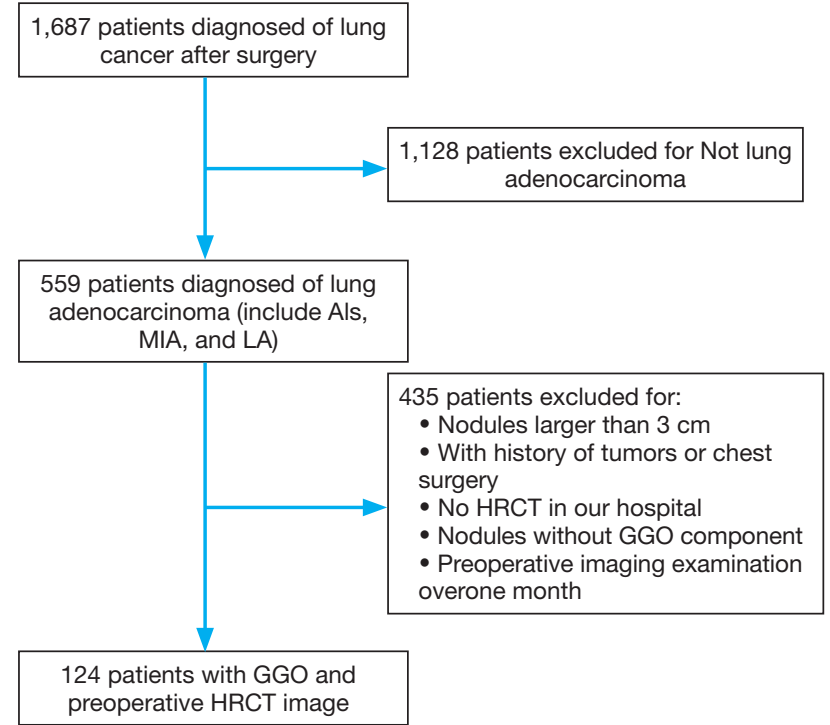

Figure 1 Selection process for patients with lung adenocarcinoma with pure or mixed ground glass opacity on high-resolution computed tomography (HRCT).

has a considerable impact on prognosis, as has been verified in many studies $(3,4)$. Most AIS and MIA cases appear as a ground glass opacity (GGO) on computed tomography (CT), whereas pure GGO nodules are less frequently associated with invasive adenocarcinoma (IA) (5). However, it is very difficult for pathologists to differentiate the pathological types on a preoperative examination such as endobronchial biopsy, fine-needle aspiration, or intraoperative frozen section (6). The consolidation tumor ratio (CTR) and maximum tumor dimension (MTD) have been used to predict tumor invasiveness and prognosis $(4,7,8)$. Lesions with MTD $<2 \mathrm{~cm}$ and CTR $<0.5$ tend to be less invasive cancers, such as AIS and MIA. However, a recent study showed that the CTR and MTD could not accurately predict the invasive status preoperatively (9).

The mean CT (m-CT) values have also been used to predict tumor invasiveness in many studies (10-12). However, there has been no study investigating the m-CT value in GGO to differentiate IA from MIA and AIS, which is of great significance in making treatment decisions. Likewise, few reports have described the relationship between histological types and CT imaging features, such as margin characteristics (smooth or spiculated), air bronchogram or vacuole sign, pleural retraction, lobulated sign, vessel convergence sign, and multiple GGOs in patients with GGO lesions.

A retrospective analysis was performed to estimate the correlation between the imaging features in high-resolution CT (HRCT) and the postoperative pathology of GGO in a Chinese population. Some independent risk factors among imaging features were investigated to establish treatment strategies for GGO nodules of the lung.

\section{Methods}

We retrospectively evaluated the clinical records of patients who underwent surgical resection at the Fourth Hospital of Hebei Medical University between 2015 and 2016. The research conformed to the Declaration of Helsinki and was authorized by the Human Ethics and Research Ethics Committee of the Fourth Hospital of Hebei Medical University. Informed consent was obtained from all patients. The inclusion criterion was a diagnosis of lung cancer after surgery, HRCT showed no mediastinal metastatic lymph nodes, the minimum diameter of GGNs is $8 \mathrm{~mm}$, CT scans performed with the same scanner and same reconstruction protocol; thin-section $1 \mathrm{~mm}$ axial lung window images in DICOM format. The exclusion criteria were tumors other than adenocarcinoma, nodules $>3 \mathrm{~cm}$, nodules without a GGO component, lack of HRCT scans obtained at our hospital, or preoperative imaging examination $>1$ month, we also excluded patients with oncologic history and lung surgery history (Figure 1).

\section{Histological evaluation}

Tumors were classified as AIS, MIA, or IA, according to the 2011 International Association for the Study of Lung Cancer/American Thoracic Society/European Respiratory Society (IASLC/ATS/ERS) classification of lung adenocarcinomas. In our study, AIS and MIA were classified in the noninvasive group and IA was classified in the invasive group. All tumors were staged using the 8th edition Union for International Cancer Control/ American Joint Committee on Cancer TNM (tumor, node, metastasis) staging system. Each specimen was evaluated by 3 pathologists with $>10$ years of experience.

\section{CT acquisition}

All patients were scanned using a dual-source CT machine (SOMATOM Definition Flash; Siemens Healthcare, Forchheim, Germany). A standard scanning protocol was applied in single mode $(64 \times 0.6 \mathrm{~mm}$ section collimation, $0.5 \mathrm{~s}$ rotation time, $120 \mathrm{kVp}$, pitch 1.0 , matrix size $512 \times 512$, 
Table 1 Clinical and pathological characteristics

\begin{tabular}{|c|c|}
\hline Variables & Total $(n=124)$ \\
\hline \multicolumn{2}{|l|}{ Age, $n(\%)$} \\
\hline$>60$ years & $49(39.5)$ \\
\hline$\leq 60$ years & $75(60.5)$ \\
\hline Median [range] & 59 [44-72] \\
\hline \multicolumn{2}{|l|}{ Gender, n (\%) } \\
\hline Male & $55(44.4)$ \\
\hline Female & $69(55.6)$ \\
\hline \multicolumn{2}{|l|}{ Smoking, n (\%) } \\
\hline Yes & $42(33.9)$ \\
\hline No & $82(66.1)$ \\
\hline \multicolumn{2}{|l|}{ Family history, $\mathrm{n}(\%)$} \\
\hline Yes & $16(12.9)$ \\
\hline No & $108(87.1)$ \\
\hline \multicolumn{2}{|l|}{ Tumor location } \\
\hline Left upper lobe & $20(16.1)$ \\
\hline Left lower lobe & $38(30.6)$ \\
\hline Right upper lobe & $26(21.0)$ \\
\hline Right middle lobe & $18(14.5)$ \\
\hline Right lower lobe & $22(17.7)$ \\
\hline \multicolumn{2}{|l|}{ Surgical procedures } \\
\hline Wedge resection & $11(8.9)$ \\
\hline Segmentectomy & $8(6.5)$ \\
\hline Lobectomy & $105(84.6)$ \\
\hline \multicolumn{2}{|l|}{ Histological subtype } \\
\hline Adenocarcinoma in situ & $20(16.1)$ \\
\hline Minimally invasive adenocarcinoma & $51(41.1)$ \\
\hline Invasive adenocarcinoma & $53(42.7)$ \\
\hline \multicolumn{2}{|l|}{ Pathologic $\mathrm{N}$ stage } \\
\hline No & $119(96.0)$ \\
\hline $\mathrm{N} 1$ & $3(2.4)$ \\
\hline N2 & $2(1.6)$ \\
\hline
\end{tabular}

and automatic exposure control (CARE Dose) with a quality reference value of $200 \mathrm{mAs}$, covering the thorax). Axial images were reconstructed by FBP with a slice thickness of $1.0 \mathrm{~mm}$ and a lung reconstruction kernel with mediastinal [level: 40 Hounsfield units (HU), width: $350 \mathrm{HU}$ ] and lung (level: $-600 \mathrm{HU}$, width: 1,200 HU) window settings.

\section{Image analysis}

Two radiologists with 10 years of experience and one thoracic surgeon with 5 years of experience retrospectively interpreted the HRCT images independently. In the case of differing opinions, a third radiologist was consulted. And we analyzed some characteristics of nodules including air bronchogram, vessel convergence sign, pleural retraction, MTD, CTR, etc. MTD was defined as the largest diameter of the lesion on the lung window setting, and the diameter of consolidation was also measured on the lung window setting. A pure GGO had a CTR of 0 , the CTR was 1 in a solid nodule. The $\mathrm{m}-\mathrm{CT}$ value was measured at the maximum diameter of the solid component.

\section{Statistical analysis}

The roles of the m-CT value, CTR, and MTD in predicting IA were compared through receiver-operating characteristic (ROC) curve analysis. Statistical analysis was performed using one-way analysis of variance (ANOVA) for continuous variables and the chi-square test or Fisher's exact test for categorical variables. We used a logistic regression model for multivariate analysis to analyze the relationship between imaging features and histological types. All statistical analyses were 2 -tailed, and $\mathrm{P}$ values $<0.05$ were deemed statistically significant. Statistical analysis was conducted using Statistical Product and Service Solutions version 22.0 (SPSS Inc., Chicago, IL, USA). The nomogram was established with R (http://www.R-project. org) and Empower Stats (www.empowerstats.com; X\&Y Solutions Inc., Boston, MA, USA) software.

\section{Results}

\section{Clinical and pathological characteristics}

The clinical features are summarized in Table 1. According to the IASLC/ATS/ERS classification, there were 20 patients with AIS (16.1\%), 51 patients with MIA (41.1\%), and 53 patients with IA $(42.7 \%)$ (Table 1$)$. There were 53 patients $(42.7 \%)$ in the invasive group and 71 patients $(57.3 \%)$ in the non-invasive group (Table 2). Lymph node metastases were detected in 5 patients, including 3 (2.4\%) with $\mathrm{N} 1$ metastasis and $2(1.6 \%)$ with $\mathrm{N} 2$ metastasis (Table 
Table 2 The association between clinical and pathological characteristics and invasive condition

\begin{tabular}{|c|c|c|c|c|c|}
\hline Factors & $\mathrm{N}$ & $\begin{array}{c}\text { Invasive } \\
(n=53)\end{array}$ & $\begin{array}{c}\text { Non-invasive } \\
\quad(n=71)\end{array}$ & $\chi^{2}$ & $P$ \\
\hline Age, n (\%) & & & & 1.195 & 0.274 \\
\hline$>60$ years & 49 & $18(34.0)$ & $31(43.7)$ & & \\
\hline$\leq 60$ years & 75 & $35(66.0)$ & $40(56.3)$ & & \\
\hline Gender, n (\%) & & & & 0.034 & 0.853 \\
\hline Male & 55 & $23(43.4)$ & $32(45.1)$ & & \\
\hline Female & 69 & $30(56.6)$ & $39(54.9)$ & & \\
\hline Smoking, $\mathrm{n}(\%)$ & & & & 0.133 & 0.715 \\
\hline Yes & 42 & $17(32.1)$ & $25(35.2)$ & & \\
\hline No & 82 & 36 (67.9) & $46(64.8)$ & & \\
\hline Family history, n (\%) & & & & 0.206 & 0.65 \\
\hline Yes & 16 & $6(11.3)$ & $10(14.1)$ & & \\
\hline No & 108 & $47(88.7)$ & $61(85.9)$ & & \\
\hline Tumor location, n (\%) & & & & 3.039 & 0.551 \\
\hline Left upper lobe & 20 & $10(18.9)$ & $10(14.1)$ & & \\
\hline Left lower lobe & 38 & $18(34.0)$ & $20(28.2)$ & & \\
\hline Right upper lobe & 26 & $8(15.1)$ & $18(25.4)$ & & \\
\hline Right middle lobe & 18 & $9(17.0)$ & $9(12.7)$ & & \\
\hline Right lower lobe & 22 & $8(15.1)$ & $14(19.7)$ & & \\
\hline \multicolumn{2}{|c|}{ Pathologic N stage, n (\%) } & & & & 0.013 \\
\hline No & 119 & $48(90.6)$ & $71(100.0)$ & & \\
\hline $\mathrm{N} 1 / \mathrm{N} 2$ & 5 & $5(9.4)$ & $0(0)$ & & \\
\hline
\end{tabular}

1). The lymph node metastatic rate was significantly higher in the invasive group (9.4\%) than in the non-invasive group $(0 \%)(\mathrm{P}=0.013)$ (Table 2).

\section{Correlation between CT characteristics and pathological subtypes}

The examples of GGO lesions are presented in Figure 2. Figure $2 A$ shows pure GGO, the m-CT value of which was $-666 \mathrm{HU}$ and the postoperative pathology was AIS. Figure $2 B$ presents mixed GGO, with IA as the postoperative pathology. Figure $2 C$ shows mixed GGO with vacuole and speculation, with a postoperative pathology of IA.

"Kolmogorov-Smirnov" in SPSS was used to test the distribution of the mean-CT value. The result showed a Non-normal distribution. From the ROC curve analysis, the cutoff value was $-410 \mathrm{HU}$ for the $\mathrm{m}-\mathrm{CT}$ value, $10 \mathrm{~mm}$ for the MTD, and $0.25 \%$ for the CTR. The area under ROC curve (AUC) value was 0.818 [95\% confidence interval (CI), 0.738-0.881] for the m-CT value, 0.697 (95\% CI, 0.608-0.776) for MTD, and 0.795 (95\% CI, $0.713-$ 0.862) for CTR (Figure 3).

The comparison of imaging features between lesions in IA and AIS or MIA are summarized in Table 3. After univariate analysis with one-way ANOVA, the significant factors including MTD, CTR, margin invasion, air bronchogram, pleural indentation, multiple GGOs, and $\mathrm{m}$-CT value were entered into multivariate analysis (Table 4). Multivariate analysis revealed that MTD [risk ratio (RR), 5.047; 95\% CI, 1.325-19.223; $\mathrm{P}=0.018$ ], air bronchogram
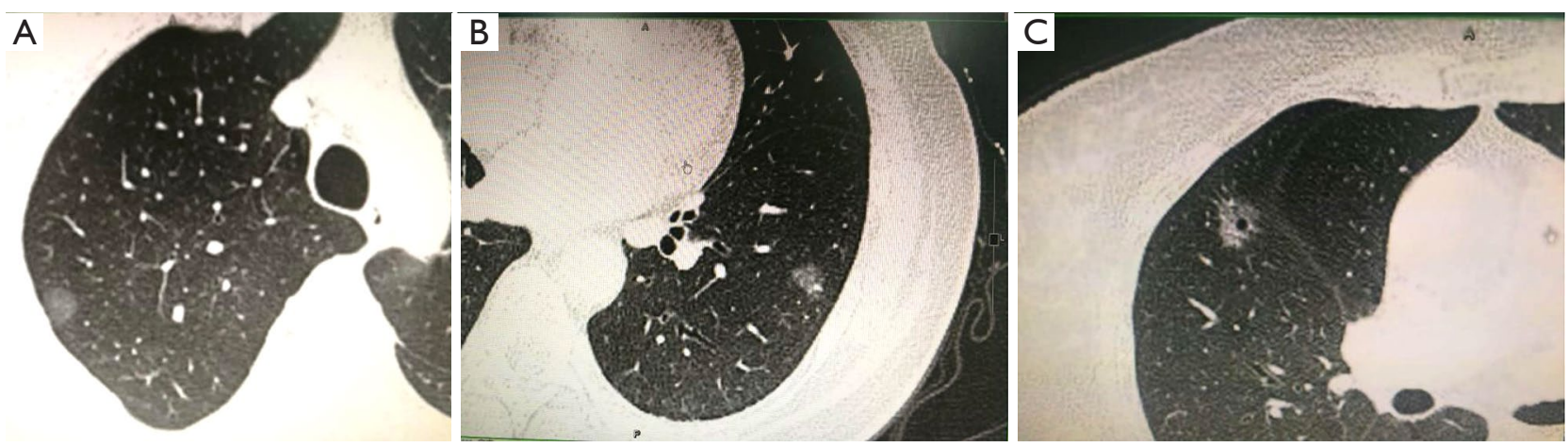

Figure 2 High-resolution computed tomography (CT) findings of typical ground glass opacity (GGO) lesions. (A) Pure GGO whose mean CT value is -666 Hounsfield units and whose postoperative pathology is adenocarcinoma in situ. (B) Mixed GGO whose postoperative pathology is invasive carcinoma (IA). (C) Mixed GGO with vacuole and speculation whose postoperative pathology is IA. 


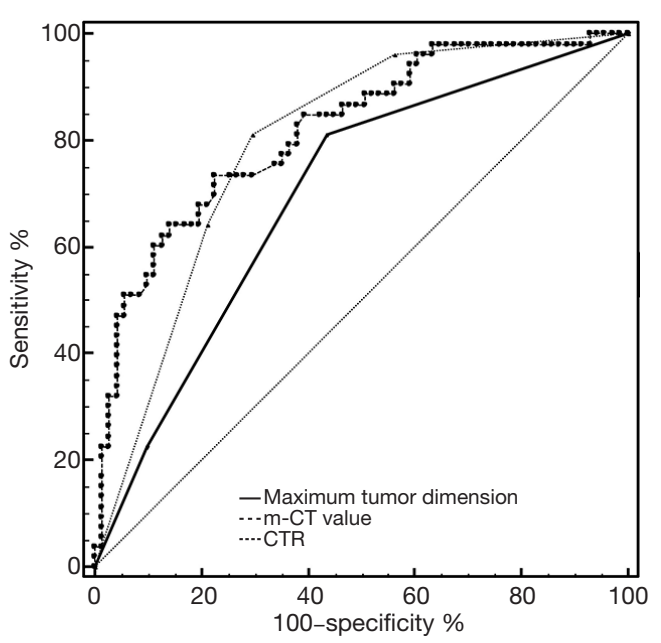

Figure 3 Receiver-operating characteristic (ROC) curves predicting less invasive cancer. CTR, consolidation tumor ratio; m-CT value, mean computed tomography value.

or vacuole sign (RR, 4.054; 95\% CI, 1.191-13.798; $\mathrm{P}=0.025)$, pleural retraction ( $\mathrm{RR}, 4.742$; $95 \%$ CI, 1.489 15.105; $\mathrm{P}=0.008)$, and $\mathrm{m}-\mathrm{CT}$ value $(\mathrm{RR}, 5.874 ; 95 \% \mathrm{CI}$, 1.716-20.101; $\mathrm{P}=0.005)$ were independent predictive factors of IA (Table 4).

\section{Nomogram and its predictive performance}

The regression coefficients ( $\beta$ ) from the multivariate logistic model were used to construct the model for estimation of the IA risk. The scoring model was as follows: $-3.50744+$ $1.26374 \times($ MTD $>10 \mathrm{~mm}=1)+2.41978 \times(\mathrm{m}-\mathrm{CT}$ value $\geq-410 \mathrm{HU}=1)+1.77779 \times($ with air bronchogram or vacuole sign $=1)+1.60913 \times($ with pleural retraction $=1)$ (Figure 4). A nomogram for predicting the probability of IA was developed using the following variables: MTD, $\mathrm{m}-\mathrm{CT}$ value, air bronchogram or vacuole sign, and pleural retraction. Each point can be established by drawing a line from each variable to the point axis, and the total point is calculated as the sum of the 4 points. Then, a line is drawn from the axis of the total points to determine the IA probabilities at the lower line of the nomogram. The performance of the nomogram was measured using ROC curves, and the AUC was 0.9 (95\% CI, 0.8482-0.9512) in the model from the observed data. The cutoff score was -0.5502 with a sensitivity of $86.8 \%$ and a specificity of $78.9 \%$ (Figure 5).

\section{Discussion}

In the present study, we retrospectively studied the HRCT features and postoperative pathological types of $\leq 3 \mathrm{~cm}$ lung nodules with GGO components. Studies have observed that women without a history of smoking account for the majority of lung adenocarcinoma, however, the results of group comparison did not show statistical differences (13). Consistent with this result, women accounted for $55.6 \%$ of the overall patients and $66.1 \%$ of nonsmokers in this study. We also tried to include gender and smoking history in a multivariate analysis, but the analysis results showed no change from the current results.

Contrary to some previous studies that combined AIS and atypical adenomatous hyperplasia into a single group and MIS and IA into another group, we categorized MIA and AIS into the noninvasive group and IA into the invasive group based on lymph node metastasis and prognosis. This grouping method is more conducive to predicting the prognosis of patients, and has more guiding value for clinical decisions. The lymph node metastatic rate was $0 \%$ in the noninvasive group and $9.4 \%$ in the invasive group. In this study, lymph node sampling was used in some cases of sublobectomy (wedge resection and segmental lung resection), which may have a certain influence on the results. The results of this study are consistent with those of some recent studies (14).

The correlations between the maximum diameter of GGO and the pathological result and between CTR and the pathological result have not been fully discussed because different centers calculate the maximum diameter and CTR of GGO with different methods. In the study conducted by Suzuki et al, 0.5 was initially set as the grouping standard for CTR, resulting in poor sensitivity. When CTR was tested at 0.25 and 0.75 , lower sensitivity and higher specificity were observed at 0.25 . Therefore, 0.25 was recommended as the cutoff value of CTR, whereas the cutoff value of the maximum diameter of the lesion was determined to be $20 \mathrm{~mm}$ (15). In the study by Tamura et al, the CTR was 0.5 and the maximum diameter was $15.6 \mathrm{~mm}$ (11). Our study suggested that the cutoff values for CTR and maximum diameter were 0.25 and $10 \mathrm{~mm}$, respectively, which differed from those in other studies because we grouped IA separately.

Previous studies focused on the correlation between CTR and MTD in GGO. Some even attempted to predict the postoperative survival of patients based on 
Table 3 The basic clinical characteristics and the situation of invasion

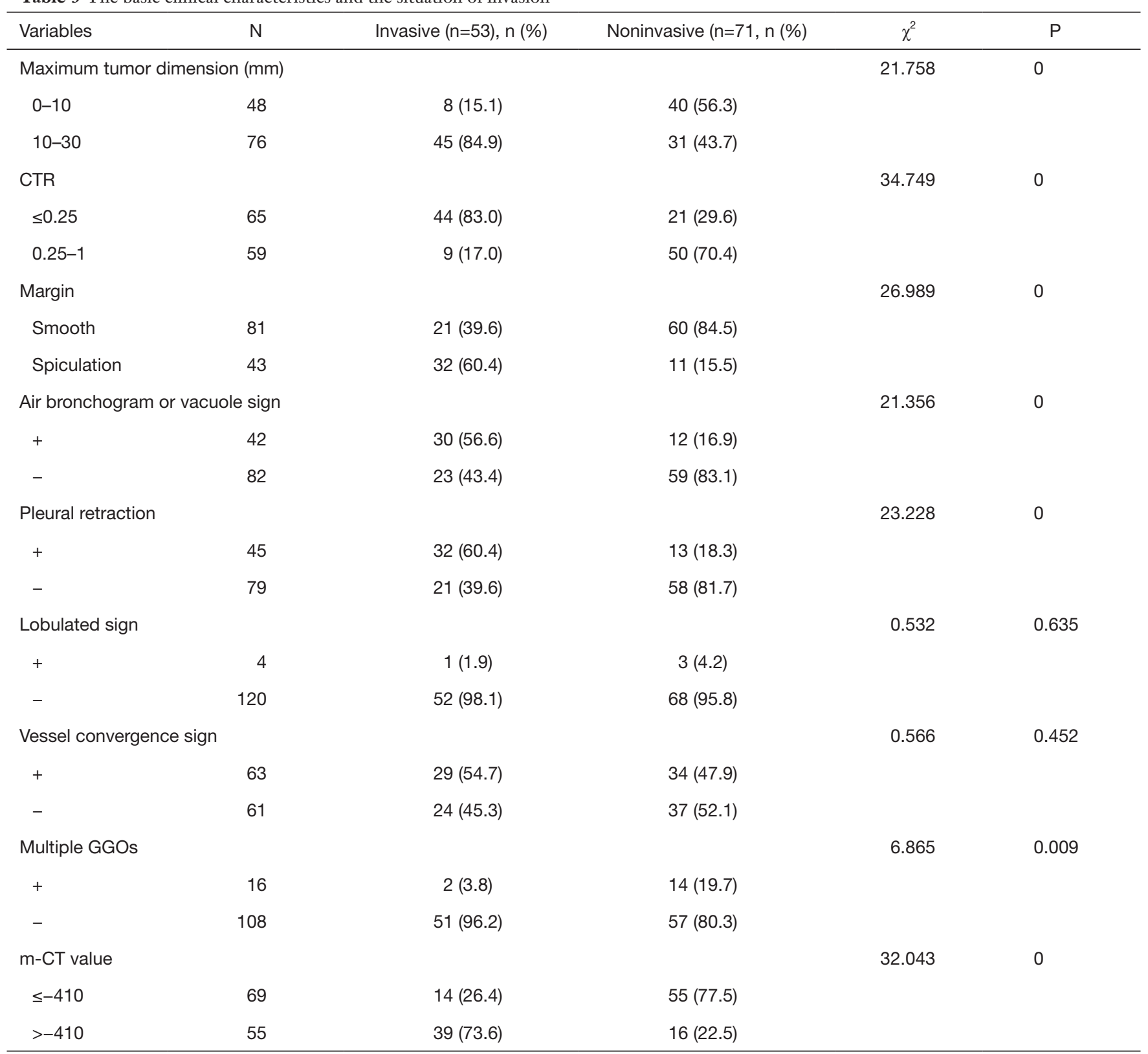

CTR and MTD. The latest study suggested that the only independent factor affecting the prognosis of patients was the infiltration status of the tumor (IA $v s$. AIS/MIA), in which IA resulted in worse lung cancer-specific overall survival than AIS and MIA (9). Some studies have suggested that $\mathrm{m}$-CT values show superior sensitivity and specificity over those of CTR and maximum diameter in predicting the pathological type of GGO. However, different studies used different measurement methods and groupings. In the study by Tamura et al., the m-CT value was measured on the layer where the lesion density was the highest. The efficiency of the m-CT value, CTR, and maximum diameter was also compared using ROC analysis. The maximum sensitivity and specificity of the $\mathrm{m}-\mathrm{CT}$ value were acquired at a cutoff value of $-445 \mathrm{HU}$, whereas the AUC of the m-CT value was 0.88 , which was higher than that in other studies (11). Peng et al. measured the m-CT value of the lesion after 3-dimensional reconstruction, and found 
Table 4 Correlation between CT characteristics and pathologic subtypes

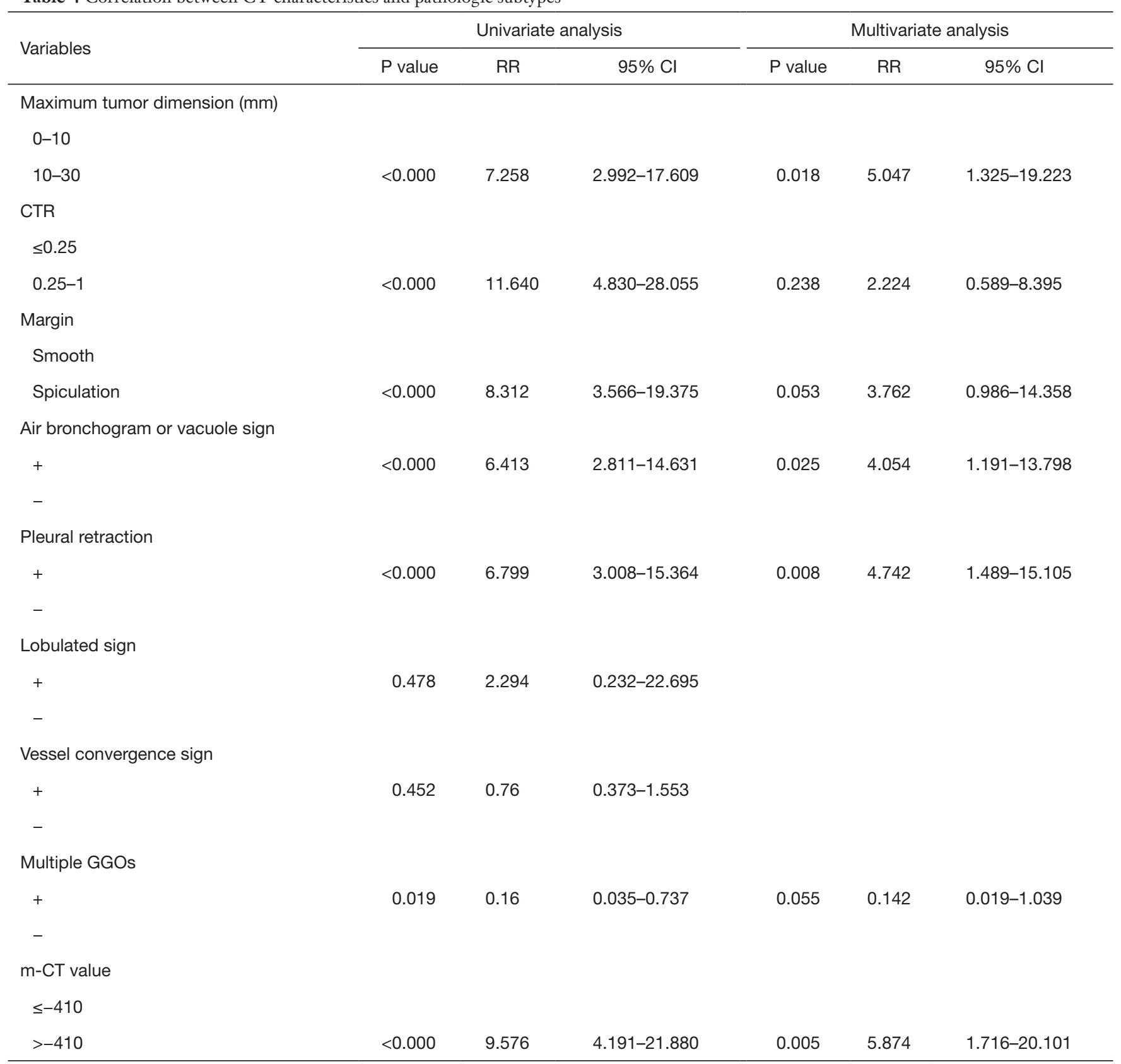

CTR, consolidation tumor ratio; RR, relative risk; $\mathrm{Cl}$, confidence interval; GGO, Ground-glass opacity; m-CT value, mean computed tomography value.

that the m-CT value has a good ability to identify MIA and AIS (16). In our study, we measured the m-CT value at the maximum diameter of the solid component, with a measured discrimination threshold of $-410 \mathrm{HU}$. According to our experience, this method is more convenient than other methods to use in different hospitals, and it is also convenient for doctor to compare the changes of GGO solid components at same patient in different time.

GGO also exhibits some conventional CT characteristics, such as pleural retraction, margin, and lobulated sign, which have been used to identify malignant lesions $(17,18)$. To achieve better prediction of GGO properties, these CT 


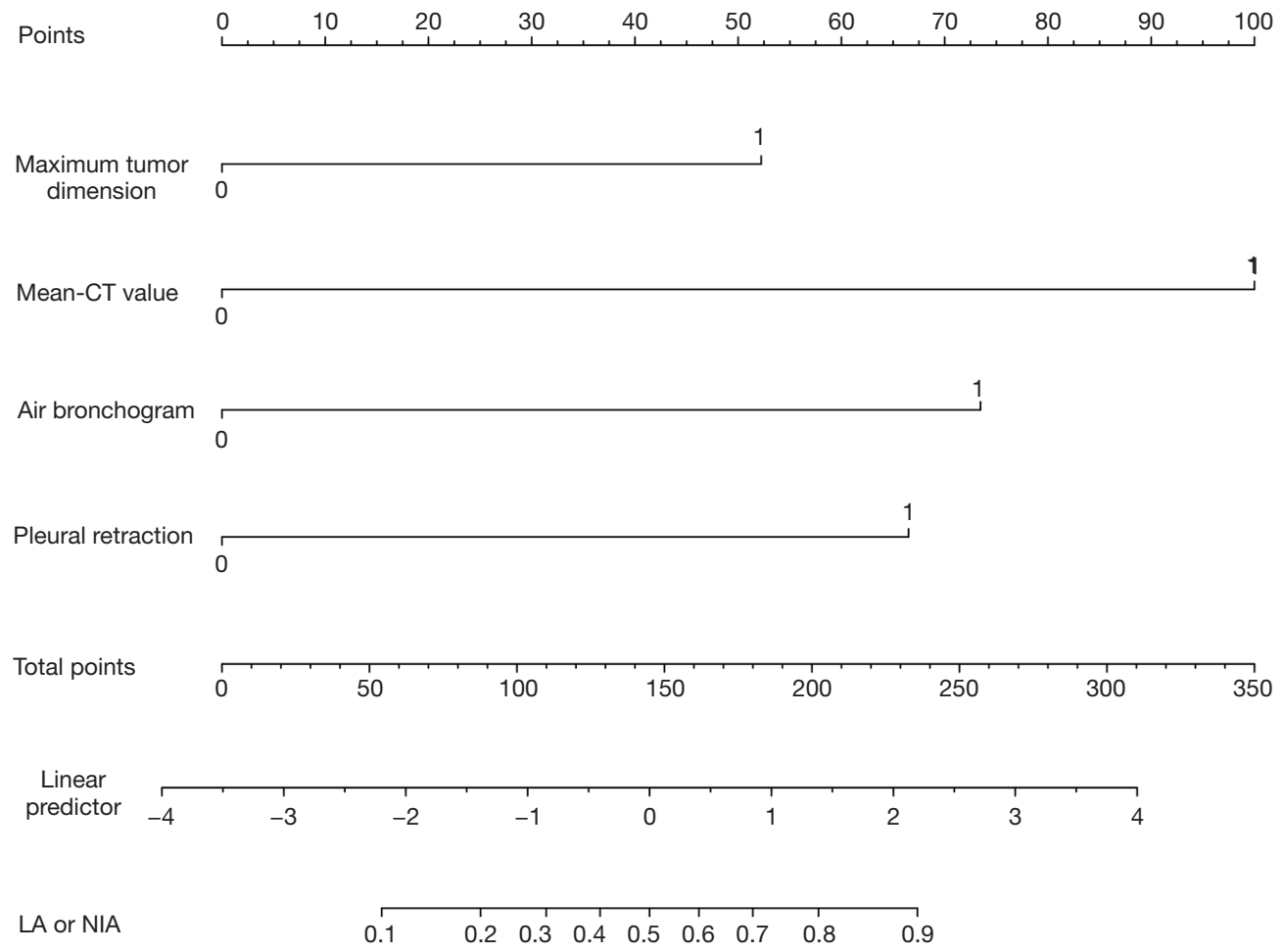

Figure 4 Nomogram for estimating the risk of invasive adenocarcinoma (IA) in patients. To use the nomogram, find the position of each variable on the corresponding axis, draw a line to the points axis of points for the number of points, add the points from all variables, and draw a line from the axis of the total points to determine the IA probabilities at the lower line of the nomogram. For example, in 1 patient with ground glass opacity (GGO) whose mean tumor dimension (MTD) is $20 \mathrm{~mm}$, mean computed tomography (m-CT) value is -210 Hounsfield units, with pleural retraction and, without air bronchogram, each score value is 52, 100, 0 , and 65 . The total score is 217 . The corresponding IA probability is about $85 \%$ in the nomogram model.

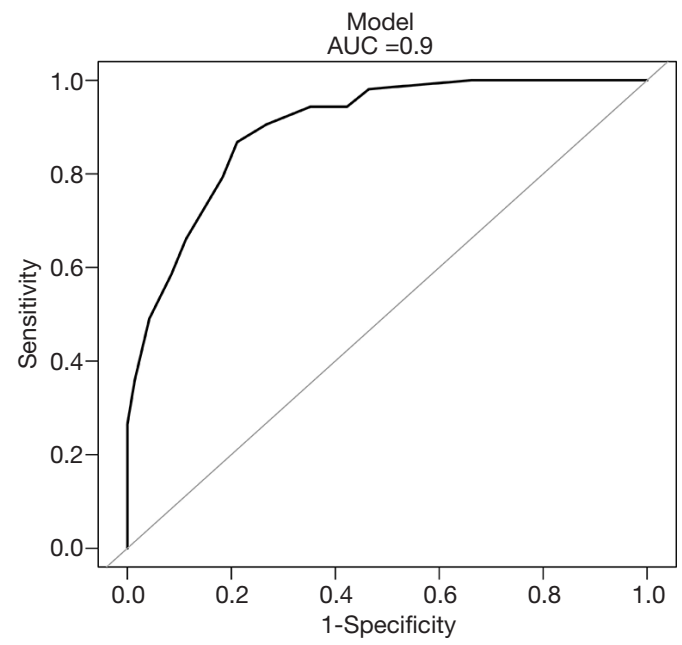

Figure 5 The AUC of the model from observed data (nomogram) was 0.9 . The cutoff score was -0.5502 with a sensitivity of $86.8 \%$ and a specificity of $78.9 \%$. AUC, area under the receiver-operating characteristic curve. signatures combined with CTR, MTD, and m-CT value were analyzed using univariate and multivariate regression. Air bronchogram, pleural retraction, maximum lesion diameter, and m-CT value were found to be independent risk factors for pathology. A novel and practical nomogram was established to accurately predict IA (AUC =0.9) in the present study. To our knowledge, this is the first report using a nomogram to predict IA. Given the impact of the pathological type of adenocarcinoma on prognosis, this study may conduct surgeons to make more accurate decisions.

However, there are some deficiencies in the research. First, during the pathogenesis of GGO, the solid components of pure GGO gradually increase, and the lesion could become mixed GGO and finally a solid nodule. The long course of GGO led to the lack of imaging data for longterm observation of lesions. If follow-up imaging data were available, time may be added as a variable to the model to improve the accuracy. Second, this study did not provide a 
more detailed stratified analysis of patients, such as patients with a smoking habit and patients with chronic lung disease, whose HRCT results may be different. We plan to include these factors in the next prospective study. Third, this is only the result of a single center study, and it is very important to perform multi-center researches in future.

\section{Conclusions}

In conclusion, a combination of $\mathrm{m}-\mathrm{CT}$ value and HRCT signatures such as MTD, CTR, margin, air bronchogram, and pleural retraction may help researchers infer the pathological type of GGO. Furthermore, a nomogram based on a combination of MTD, m-CT value, air bronchogram, and pleural retraction may accurately predict IA in patients with GGO lesions.

\section{Acknowledgments}

Funding: None.

\section{Footnote}

Conflicts of Interest: All authors have completed the ICMJE uniform disclosure form (available at http://dx.doi. org/10.21037/tcr.2020.01.55). The authors have no conflicts of interest to declare.

Ethical Statement: The authors are accountable for all aspects of the work in ensuring that questions related to the accuracy or integrity of any part of the work are appropriately investigated and resolved. The research conformed to the Declaration of Helsinki (as revised in 2013) and was authorized by the Human Ethics and Research Ethics Committee of the Fourth Hospital of Hebei Medical University. Informed consent was obtained from all patients.

Open Access Statement: This is an Open Access article distributed in accordance with the Creative Commons Attribution-NonCommercial-NoDerivs 4.0 International License (CC BY-NC-ND 4.0), which permits the noncommercial replication and distribution of the article with the strict proviso that no changes or edits are made and the original work is properly cited (including links to both the formal publication through the relevant DOI and the license). See: https://creativecommons.org/licenses/by-nc-nd/4.0/.

\section{References}

1. Miao Y, Zhang J, Zou J, et al. Correlation in histological subtypes with high resolution computed tomography signatures of early stage lung adenocarcinoma. Transl Lung Cancer Res 2017;6:14-22.

2. Travis WD, Brambilla E, Noguchi M, et al. International association for the study of lung cancer/american thoracic society/european respiratory society international multidisciplinary classification of lung adenocarcinoma. J Thorac Oncol 2011;6:244-85.

3. Murakami S, Ito H, Tsubokawa N, et al. Prognostic value of the new IASLC/ATS/ERS classification of clinical stage IA lung adenocarcinoma. Lung Cancer 2015;90:199-204.

4. Takahashi M, Shigematsu Y, Ohta M, et al. Tumor invasiveness as defined by the newly proposed IASLC/ ATS/ERS classification has prognostic significance for pathologic stage IA lung adenocarcinoma and can be predicted by radiologic parameters. J Thorac Cardiovasc Surg 2014;147:54-9.

5. Moon Y, Sung SW, Lee KY, et al. Pure ground-glass opacity on chest computed tomography: predictive factors for invasive adenocarcinoma. J Thorac Dis 2016;8:1561-70.

6. Xu X, Chung JH, Jheon S, et al. The accuracy of frozen section diagnosis of pulmonary nodules: evaluation of inflation method during intraoperative pathology consultation with cryosection. J Thorac Oncol 2010;5:39-44.

7. Suzuki S, Sakurai H, Yotsukura M, et al. Clinical Features of Ground Glass Opacity-Dominant Lung Cancer Exceeding $3.0 \mathrm{~cm}$ in the Whole Tumor Size. Ann Thorac Surg 2018;105:1499-506.

8. Hattori A, Takamochi K, Oh S, Suzuki K. Prognostic classification of multiple primary lung cancers based on a ground glass opacity component. Ann Thorac Surg 2020;109:420-7.

9. Ye T, Deng L, Xiang J, et al. Predictors of Pathologic Tumor Invasion and Prognosis for GGO Featured Lung Adenocarcinoma. Ann Thorac Surg 2018;106:1682-90.

10. Kitazawa S, Saeki Y, Kobayashi N, et al. Threedimensional mean CT attenuation value of pure and partsolid ground-glass lung nodules may predict invasiveness in early adenocarcinoma. Clin Radiol 2019;74:944-9.

11. Tamura M, Matsumoto I, Saito D, et al. Mean Computed Tomography Value to Predict the Tumor Invasiveness in Clinical Stage IA Lung Cancer. Ann Thorac Surg 2017;104:261-6. 
12. Lee HY, Choi YL, Lee KS, et al. Pure ground-glass opacity neoplastic lung nodules: histopathology, imaging, and management. AJR Am J Roentgenol 2014;202:W224-33.

13. Moon Y, Park JK, Lee KY, Ahn S, Shin J. Predictive factors for invasive adenocarcinoma in patients with clinical non-invasive or minimally invasive lung cancer. J Thorac Dis 2018;10:6010-9.

14. Hashizume T, Yamada K, Okamoto N, et al. Prognostic significance of thin-section CT scan findings in small-sized lung adenocarcinoma. Chest 2008;133:441-7.

15. Suzuki K, Koike T, Asakawa T, et al. A prospective radiological study of thin-section computed tomography to predict pathological noninvasiveness in peripheral clinical
IA lung cancer (Japan Clinical Oncology Group 0201). J Thorac Oncol 2011;6:751-6.

16. Peng M, Peng F, Zhang C, et al. Preoperative Prediction of Ki-67 Labeling Index By Three-dimensional CT Image Parameters for Differential Diagnosis Of Ground-Glass Opacity (GGO). PLoS One 2015;10:e0129206.

17. Toyokawa G, Yamada Y, Tagawa T, et al. Computed tomography features of resected lung adenocarcinomas with spread through air spaces. J Thorac Cardiovasc Surg 2018;156:1670-6.e4.

18. Dai J, Yu G, Yu J. Can CT imaging features of groundglass opacity predict invasiveness? A meta-analysis. Thorac Cancer 2018;9:452-8.
Cite this article as: Zhang N, Liu JF, Wang YN, Yang L. A nomogram to predict invasiveness in lung adenocarcinoma presenting as ground glass nodule. Transl Cancer Res 2020;9(3):1660-1669. doi: 10.21037/tcr.2020.01.55 